\title{
Comparing the Effectiveness Between Gel and Foam Hand Sanitizers
}

Caroline Leung ${ }^{1}$, Helen Heacock ${ }^{2}$, Tina Chen $^{3}$

1 Lead Author, B. Tech Student, School of Health Sciences, British Columbia Institute of Technology, 3700 Willingdon Ave, Burnaby, B.C., V5G 3H2

2 Supervisor, School of Health Sciences, British Columbia Institute of Technology, 3700 Willingdon Ave, Burnaby, B.C., V5G 3H2

3 Contributor, National Collaborating Center for Environmental Health, 200 - 601 West Broadway, Vancouver, B.C., V5Z 4C2

\begin{abstract}
Background: Hand sanitizers are commonly used as an alternative to washing hands with warm water and soap. There are a variety of different hand sanitizers including gel and foam and they are known to kill several bacteria. Many factors play a role in the effectiveness of hand sanitizers such as the alcohol concentration and the techniques used to apply hand sanitizers. Alcohol based hand sanitizers must have an alcohol concentration of $60-70 \%$ to be effective. There is currently no legislation regulating hand sanitizers and there is a lack of research focusing on differences between foam and gel hand sanitizers. This research study investigates effectiveness of gel compared to foam hand sanitizers by evaluating the prevalence of Escherichia coli (E.coli) on pigskins.
\end{abstract}

Methods: To compare the hand sanitizers, microbiological sampling was completed. E.coli was introduced onto 65 pigskins. Five pigskins were used as a baseline to determine the average amount of Colony Forming Units (CFUs) of E.coli present prior to the application of hand sanitizers. One set of the 30 pigskins was applied with gel hand sanitizer, whereas the other 30 was applied with foam hand sanitizer. The pigskins were swabbed with QuickSnap swabs and plated onto $3 \mathrm{M}$ Petrifilms. The 65 petrifilms were incubated at $35^{\circ} \mathrm{C}$ for 48 hours. After incubation, the CFUs of E.coli present on the petrifilm were enumerated. The difference in CFUs was calculated to determine the reduction in E.coli and the overall effectiveness of hand sanitizers.

Results: The data was analyzed by using the statistical software, NCSS. Statistical analysis showed that the findings were statistically significant and the null hypothesis (Ho: no difference in CFUs of E.coli between foam versus gel alcohol-based hand sanitizers) was rejected with a power of 0.9997 at $p$-value of 0.00000 . This indicates that there is a difference in the ability to reduce E.coli between gel and foam hand sanitizers and gel sanitizers appeared to be more effective.

Conclusion: These results indicate that there was a difference in the effectiveness between foam and gel hand sanitizers in reducing E.coli that was inoculated onto pigskins. However, consumers should be aware that hand sanitizers do not completely eliminate all pathogens. Though gel hand sanitizers are more effective, they should only be used when there are no other methods of keeping hands clean.

Keywords: E.coli, Escherichia coli, Foam, Gel, Hand Sanitizer, Pigskin, Public health 


\section{Introduction}

It has been observed that there is an increased use of hand sanitizers in healthcare settings such as hospitals. The easy application of hand sanitizers allows for the increase in compliance among users as they are known to prevent the spread of diseases (Pickering et al, 2013). Companies such as Purell advertise that hand sanitizers "kill $99.99 \%$ of the most common germs that may cause illness" (Purell, 2015). Although hand sanitizers are commonly used as a quick and easy alternative to hand washing, they should not replace traditional hygienic practices. Thus, hand sanitizers should be only be used as an alternative when there are no opportunities to access potable water or to wash hands with soap and warm water (CDC, 2015).

There are a variety of hand sanitizers sold in retail stores and this includes foam and gel hand sanitizers. Studies have been done on liquid hand sanitizers but there is a knowledge gap in terms of comparing the effectiveness between these different consistencies. Deriving from the author's curiosity and interest in the increased usage of hand sanitizers, it would be beneficial to assess the effectiveness of different types that are commercially available.

\section{Literature Review}

\section{Types of Hand Sanitizers}

Hand sanitizers can be described as

"an alternative to handwashing with soap that do not require water" (Pickering et al, 2011). Different types of hand sanitizers are composed of different active ingredients.

There are two types: alcohol-based and nonalcohol based. Alcohol-based hand sanitizer products are known to contain a specific percentage of alcohol such as "ethanol, npropanol, or isopropanol” (Patel, 2004). Non-alcohol based hand sanitizers generally contain a chemical compound, benzalkonium chloride (SafeHands, 2015).
In addition, hand sanitizers can be further categorized by their consistency: liquid, gel, and foam (Purell, 2015).

\section{Efficacy of Alcohol-based Hand Sanitizers}

Efficacy of hand sanitizers depends

on various factors including the alcohol concentration, the application techniques, and the amount used. In order to achieve proper sanitation of hands, Reynolds et al (2006) states that "educational efforts should emphasize that effective sanitizers must be of a sufficient alcohol concentration".

Hence, there is an association between the effectiveness of sanitizers and their alcohol content. Studies show that the alcohol based hand sanitizers that contain at least $70 \%$ alcohol (such as ethanol) should be used because it is most effective (Edmonds et al, 2011). Furthermore, another study demonstrated that hand sanitizers with less than $40 \%$ alcohol content were not as effective compared to those with $60 \%$ alcohol (Reynolds et al, 2006). The remaining bacterial counts for hand sanitizers with less than $40 \%$ and $60 \%$ alcohol content were relatively the same prior to the application of hand sanitizers (Reynolds et al, 2006). In addition, hand sanitizers that contained greater than $70 \%$ alcohol were not required to meet hand hygiene sanitizer written standards (Edmonds et al, 2011). Thus, in order for hand sanitizers to be effective, the alcohol concentration should be in the range of $60 \%$ to $70 \%$.

In addition to the alcohol concentration, efficacy also depends on the proper techniques used to apply hand sanitizers. As a guideline, Babeluk et al (2014) used the standard, European Norm (EN) 1500. EN 1500 indicates the methods and equipment necessary to decrease the amount of bacterial flora found on hands by using hygienic hand rubs (Babeluk et al, 2014). Babeluk et al (2014) states that the 
participants were "educated about correct hand disinfection procedures according to EN 1500" as it is illustrated in Figure 1. Through proper education, there were noticeable reduction in pathogens on participants' hand (Babeluk et al, 2014).

Rather than using EN 1500 standards as a guideline, Kampf et al (2010) ensured both of the participants' hands were completely covered with hand sanitizer. However, in each study, there was still a decrease in the amount of bacteria present on the participants' hands regardless of the application techniques.
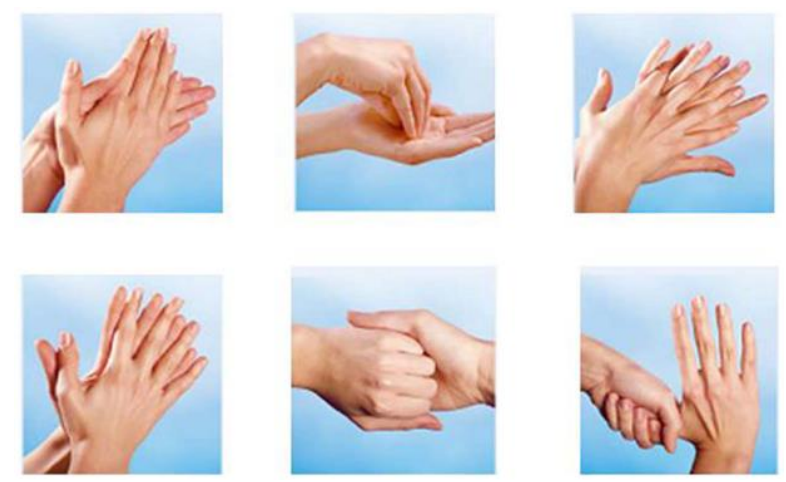

Figure 1: Hand disinfection steps in regards to EN1500 (Babeluk et al, 2014)

\section{Public Health Significance}

The public health significance of hand hygiene is to prevent the spread of diseases as "it prevents transmission of pathogens by contact and the fecal-oral route" (Widmer, 2000). Hand sanitizers are commonly found in healthcare settings but they have been observed in various locations such as malls and portable toilets. In addition, they now are packaged into small portable containers which allows for convenience when travelling. This reinforces the viewpoint that "hand sanitisers should always be openly available in public areas" (Barratt, 2015). With the easy application of hand sanitizers, there has been an increase in compliance of its usage which in turn, decreases in the spread of diseases (Pickering et al, 2013). However, the effectiveness of hand sanitizers depends on several factors such as the alcohol concentration present and the techniques used to apply hand sanitizers.

There are a variety of microorganisms found in human feces but the most commonly found pathogen is E.coli (WHO, 2016). More specifically, pathogenic E.coli can survive on human skin for more than an hour (Kampf \& Kramer, 2004). As a result of poor hand hygiene practices, E.coli can cause foodborne illnesses and outbreaks (Edmonds et al, 2012). Infected individuals would have symptoms of diarrhea and abdominal pains (WHO, 2016). Although related E.coli illnesses can be self-limiting, there are other health effects such as hemolytic uremic syndrome (HUS) that follows as a sequela (WHO, 2016). Moreover, WHO (2016) identifies that "up to $10 \%$ of patients with [E.coli] infection may develop HUS". HUS is a condition that can lead to anemia, kidney failure, and death (National Kidney Foundation, 2015). The main symptom of HUS is diarrhea (National Kidney Foundation, 2015). Thus, individuals who have direct or indirect contact with high risk individuals should be aware of their hand hygiene practices to prevent the transmission of diseases. More specifically, E.coli is often related to crosscontamination during outbreaks (Kampf \& Kramer, 2004).

\section{Legislation}

Currently, in Canada, there is no legislation, regulation or policy implemented to regulate the use of hand sanitizers. Though there are standards such as EN 1500 , it is simply used as a guideline and as well, only for in-vivo experiments. Thus, the usage of hand sanitizers cannot be regulated nor be enforced in healthcare settings which generally deals with high risk immunocompromised individuals. 


\section{Strengths and Limitations}

Strengths of the literature review includes the ability for each study to obtain access to resources. For example, Reynolds et al (2006) was able to supply written surveys prior to and after their microbiological experiments. This enabled them to understand the participants' level of knowledge before and after the study. In addition, the studies completed are replicable because the experiments were standardized and were performed in a controlled laboratory setting.

The studies performed were relatively similar and so, the limitations of the studies had a similar trend. There was contradicting information between British Columbia Centre for Disease Control (BCCDC), World Health Organization (WHO) and Pickering et al's article. BCCDC (2016) states that hand sanitizers should only be used when hands are not visibly soiled. More specifically, WHO (2009) states that hand sanitizers are not recommended for use when hands are "contaminated with proteinaceous materials". However, Pickering et al's (2011) demonstrated that there were no differences in efficacy of bacterial counts between soiled and unsoiled hands. However, the opposing arguments may be due to the fact that Pickering et al's study was completed within a controlled laboratory environment (2011). In addition, other studies used a similar artificial laboratory setting and this was not representative of the natural environment. In reality, hands come into contact with various objects which consists of numerous bacteria as Widmer (2000) describes that "the normal human skin is colonized with multiple species of microorganisms".

As well, for different studies, each individually focused on different types of microorganisms. Luby et al (2010) tested for the pathogen, C.perfringens whereas
Pickering et al (2011) tested for E.coli 0157:H7, a food-related microorganism that is most commonly found in human feces. Consequently, by doing so, it is difficult to determine the efficacy of hand sanitizers because only one type of indicator bacterium was tested rather than multiple of different bacterium as this is more representative of what is naturally found on hands.

There is a noticeable gap in research as there were a limited number of articles that specifically focused on both foam and gel hand sanitizers. However, most articles only focused on the difference between alcohol based and non-alcohol based hand sanitizers. Furthermore, each study utilized a quantitative and controlled volume of hand sanitizers. However, in actuality, it is difficult to monitor and control the amount an individual would generally apply to their own hands. Thus, the studies do not reflect real life cases. Similarly, the technique of applying hand sanitizers vary as people do not always apply hand sanitizers in the similar fashion described in EN 1500 standards.

In terms of knowledge gap, there is a common misconception that hand sanitizers can be used in replacement of the traditional practice of washing hands with warm water and soap. The increase in compliance of using hand sanitizers is beneficial. However, it is not necessarily advantageous for high risk groups or immunocompromised individuals. This is illustrated by Vidyashankar et al (2011) who believes that if the hand sanitizers are not "potent enough", there will be "pathogenic remnants or pathogen-associated molecular patterns" that can still cause harm when ingested.

\section{Purpose of the Project}

It has been demonstrated that good hand hygiene practice is associated with a decreased spread of diseases (WHO, 2013). Consequently, there has been an increased 
use of hand sanitizers as an alternative to washing hands with soap and warm water (Patel, S, 2004). With the increased use of hand sanitizers, a variety of commercially available hand sanitizers are being marketed. However, it is currently unknown if there is a significant difference in effectiveness between using one type of hand sanitizer over another. Thus, the purpose of this research study was to compare the effectiveness of gel in comparison to foam alcohol-based hand sanitizers by evaluating the prevalence of E.coli on pigskins after these two types of hand sanitizers have been applied. By performing a microbiological test, Colony Forming Units (CFUs) of E.coli were enumerated and compared between the two different types of hand sanitizers.

\section{Methods and Materials}

In this experiment, a microbiological test was performed with alcohol-based hand sanitizers of both foam and gel. A total of 60 pigskins were inoculated with an E.coli culture; 30 of which were applied with foam hand sanitizers and the other 30 with gel hand sanitizers. The prevalence of E.coli was enumerated by counting CFUs from the 60 pigskin samples. In order to compare the effectiveness of the two hand sanitizers, the difference in CFUs was determined by subtracting the remaining CFUs from the initial CFUs found on the pigskin. The initial number of CFUs was determined by inoculating five pigskins. These pigskins were swabbed with a QuickSnap swab, and placed on a $3 \mathrm{M}$ petrifilm. An average number of CFUs was obtained from these five pigskins (i.e. baseline).

Prior to the main experiment, there were two procedures that were completed. This included determining the necessary dilution factor for the E.coli solution and the preparation of pigskins. An E.coli culture solution was prepared so the surface of pigskins can be applied with E.coli (Yip,
2003). In addition, the pigskins were cut into sizes of $10 \mathrm{~cm}$ by $5 \mathrm{~cm}$ in order to provide a representative surface area.

Once the serial dilution steps were performed, the experiment involved applying the prepared E.coli solution onto 60 pigskins (Yip, 2003). Then, either foam or gel hand sanitizer was applied onto the pigskin. Again, the pigskin was swabbed but this time, it determined the remaining CFUs of E.coli present on the pigskin. The swabbed samples were placed on $3 \mathrm{M}$ petrifilm so they could be enumerated (Figure 2). From the data collected, the effectiveness of the two different hand sanitizers, foam and gel were compared. This experiment's standard method was adopted from a previous study completed by Sophia Yip in 2003.

The data collected were in CFUs which are whole numbers and thus, are discrete numerical data (Heacock, H. \& Karakilic, V., 2015b). The enumerated CFUs of E.coli were collected and entered into Microsoft Excel. The collected data was analyzed using a statistical software, NCSS.

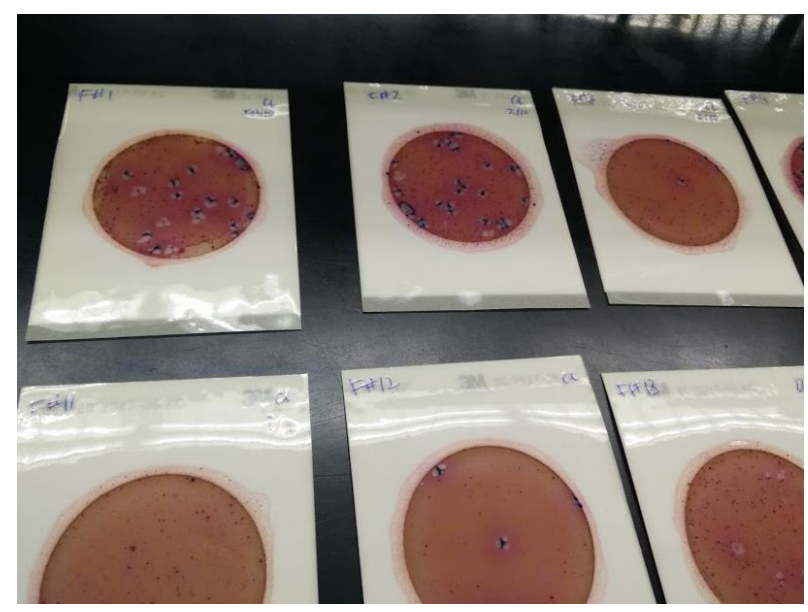

Figure 2: CFUs of E.coli on 3M petrifilm

\section{Results \\ Descriptive Statistics}

The collection of data is comprised of two parts. First, it involved counting 
CFUs of E.coli after the inoculation with E.coli but before applying the hand sanitizer. The average number of CFUs between the five pigskins (baseline) was determined to be the baseline for the initial CFUs of E.coli. Secondly, the remainder CFUs of E.coli after the application of the hand sanitizer were counted. In order to compare the effectiveness of the two hand sanitizers, the remaining CFUs of both foam and gel hand sanitizers were compared.

From the 30 samples collected of both foam and gel hand sanitizers, the means of "the difference" were 8 CFUs and 0.8 CFUs, respectively. The standard deviation of foam and gel were 1.27 and 0.33 , respectively. This is illustrated in Table 1.

\begin{tabular}{|l|l|l|}
\hline & Foam & Gel \\
\hline Mean & 8 CFU & 0.8 CFU \\
\hline $\begin{array}{l}\text { Standard } \\
\text { Deviation }\end{array}$ & 1.27 & 0.33 \\
\hline
\end{tabular}

Table 1: Mean and Standard Deviation Comparison

\section{Inferential Statistics}

Two-tailed t-test was performed to determine if there was a significant difference between the effectiveness of foam and gel hand sanitizers. The hypotheses of this study were:

Null hypothesis: There is no difference in CFUs of E.coli between foam versus gel alcohol-based hand sanitizers.

Alternative hypothesis: There is a difference in CFUs of E.coli between foam versus alcohol-based gel hand sanitizers. Initially, a normality test (test of assumption) was conducted to determine if a parametric or non-parametric test should be used (Heacock, H. \& Karakilic, V., 2015a). The Test of Assumption indicated that the data was not normally distributed, thus a non-parametric test was used (MannWhitney U or Wilcoxon Rank-Sum test). This test indicated that the $\mathrm{p}$-value was
0.00000 and thus, $H_{o}$ was rejected and it was concluded that there was a difference when comparing the effectiveness between foam versus gel hand sanitizers. Gel hand sanitizers are superior to foam hand sanitizers at reducing E.coli from pigskins.

\section{Alpha/Beta Errors and Power of Study}

$\mathrm{P}$-value was 0.00000 and hence, there is no possibility of either alpha or beta error. With a $99.9 \%$ power, it indicates that there is a difference in the ability to reduce E.coli between gel and foam hand sanitizers.

\section{Discussion}

This study successfully determined the effectiveness of gel in comparison to foam alcohol-based hand sanitizers by evaluating the presence of E.coli on pigskins after these two types of hand sanitizers were applied. Based on the study, the results indicated that gel hand sanitizer was more effective than foam hand sanitizer in reducing the number of CFUs of E.coli. The mean of foam hand sanitizer illustrated that it had ten times the number of E.coli remaining in comparison to gel hand sanitizer.

This study had distinct differences in comparison to a previous study. Although the previous study focused specifically on soap products, the consistency of the foam and liquid products were the same. The previous study suggested that the consistency of foam was more effective than liquid (Choi, 2007). The contrasting difference may arise from using different pathogens for the experiment as E.coli may be more resistant than L.acidophilus. As well, Choi (2007) stated that it was less expensive to buy foam hand sanitizer in comparison to gel hand sanitizer. However, the cost for foam hand sanitizers was more expensive than for gel hand sanitizers. Reasons for the cost difference may be due to the increase percentage of alcohol within 
the product and as well, the cost of the pump used.

For this study, there were noticeable differences between the two types of hand sanitizers and the remaining CFUs of E.coli present on the petrifilm. For pigskins that had the application of gel hand sanitizer, the remaining E.coli on the petrifilm was determined to range between 0-8 CFUs. However, for the foam hand sanitizer, the remaining E.coli ranged between 0-20 CFUs. The number of CFUs of E.coli remaining is significant because it does not support the company's claim that hand sanitizers "effectively kills $99.9 \%$ of most common disease-causing germs" (One Step, 2016). It is illustrated that many samples had only reduced at least $50 \%$ of E.coli. Choi (2007) states that it is practically impossible to completely remove all pathogens present on hands. The baseline of E.coli used for this experiment was $21 \mathrm{CFUs}$ and this is equivalent to $21,000 \mathrm{CFUs}\left(21 \times 10^{3}\right)$ per $50 \mathrm{~cm}^{2}$. Thus, One Step's claim is still valid because in reality, it is unlikely that an individual's hand would normally contain extremely large number of E.coli.

The main active ingredient for hand sanitizers is ethyl alcohol. Foam hand sanitizer has a concentration of $70 \%$ whereas gel hand sanitizers have a concentration of $62 \%$. Edmonds et al (2011) stated that hand sanitizers are most effective when the alcohol concentration ranges between 60 to $70 \%$. More specifically, he indicated that there is no noticeable difference in the reduction of pathogens when the percentage of alcohol falls between this range (Edmonds et al, 2011). Even though the foam hand sanitizer had a greater alcohol concentration than the gel hand sanitizer, the foam hand sanitizer still remained less effective. Thus, the differences in the alcohol concentration was not reflective of the product's efficacy.
As a result of poor hand hygiene practices, E.coli can be transmitted through the ingestion of contaminated foods (Edmonds et al, 2012). As well, there are chronic health effects associated with E.coli such as hemolytic uremic syndrome (WHO, 2016). Currently, there is no predetermined threshold as to how much E.coli is considered unsafe on hand surfaces. On a regular basis, it is difficult to determine the types of pathogens that are found on hands. This is further supported by Widmer (2000) who indicated that hands consist of countless numbers of pathogens. Nonetheless, the developed guideline of EN 1500 should be recognized as it ensures proper hand disinfection procedures (Babeluk et al, 2014). Thus, the public should be familiar with the proper procedures and as well, the volume of hand sanitizer that is required during its application.

\section{Recommendations}

For this study, large amounts of E.coli were inoculated onto a small surface area. It is important to acknowledge that realistically, there would not be an enormous amount of E.coli on an individual's hand. As a recommendation, one must keep in mind that the amount and as well, the type of pathogens could vary greatly on an individual's hand. From a health perspective, hand sanitizers aid in promoting hand hygiene practices as Yip (2003) indicates that One Step gel hand sanitizer was more effective than traditional hand washing practices with water and liquid soap in removing E.coli. Thus, it is beneficial that gel hand sanitizers continue to be easily accessible to the public. Through this study's findings, the use of gel hand sanitizers would be advantageous for quick and easy removal of E.coli especially in settings such as daycares and hospitals. 


\section{Limitations}

The cost was a limitation for this study. In order to meet budget constraints, the experiment was completed in collaboration with another researcher as the raw data was shared. During the experiment, it was difficult to ensure that the same techniques were used for the application of the gel hand sanitizer and as well, the swabbing methods used on the pigskins.

Each pigskin varied as there were differences in its thickness and texture. The thickness of the pigskin affected the time it required for the hand sanitizers to air dry. In addition, the texture differed because some pigskins were not bought on the same day. Another constraint was that it was difficult to ensure that both the E.coli culture and the hand sanitizers were evenly applied onto each pigskin. The amount of pressure that was applied when swabbing the sample could have greatly varied and this would have affected the amount of E.coli that was picked up by the swab.

There was also a recognizable difference between the volumes of hand sanitizer per pump. Foam hand sanitizers are known to produce foam bubbles when dispensed from the bottle and thus, the number of foam bubbles would vary for each application. As well, during the application of the foam hand sanitizer onto the pigskin, it was difficult to completely get rid of the foam bubbles. Thus, more time was necessary for the foam hand sanitizer to air dry. This could have affected the contact time of the E.coli with the hand sanitizer.

For several samples, it was also noted that there were red dots on the petrifilm. This indicates that there was a presence of total coliforms growing other than E.coli (blue dots with gas bubbles). The observed total coliforms were unquantifiable because there was an overgrowth of pathogens. The presence of total coliforms is significant because it indicates that the pigskins were not properly washed prior to the experiment. According to Pickering et al (2011), there should be no differences in efficacy of bacterial counts between soiled and unsoiled hands. On the other hand, BCCDC (2016) states that hand sanitizers should only be used when hands are not visibly soiled. Although it is difficult to distinguish what is considered to be true, the overgrowth of total coliform counts could potentially have an effect on the CFUs of E.coli present.

\section{Future Research Suggestions}

There are several future research suggestions that would ensure consistency throughout the study. In order to guarantee a more consistent texture to swab, another surface or object that simulates the texture of hands should be used. As well, a more consistent technique is necessary for the application of hand sanitizers onto pigskins. The experiment should be performed by the same researcher to increase the validity of the study. For hand sanitizers, a method should be developed to take into consideration the production of foam bubbles. So, this will determine that exactly the same volume was dispensed by both types of hand sanitizers. Although $70 \%$ ethyl alcohol foam hand sanitizers and $62 \%$ ethyl alcohol gel hand sanitizer were used, future studies should experiment with hand sanitizers containing the same percentage of alcohol.

Moreover, future studies can attempt to apply hand sanitizers on visibly soiled surfaces to determine if there is a difference in effectiveness. As well, rather than using E.coli, another pathogen could be used to determine the efficacy of hand sanitizers.

\section{Conclusion}

The findings of this study illustrate that gel hand sanitizer is more effective than foam hand sanitizer in reducing E.coli 
inoculated onto pigskins. Although the study shows that gel is more effective, it is critical that consumers should be aware of the volume of hand sanitizer that is being applied on their hands. Thus, if hand sanitizers should be used, consumers should ensure that their hands are applied with generous amounts in order to eliminate any E.coli that could be present. The outcome from the application of hand sanitizers could vary greatly since some samples did not have any CFUs of E.coli remaining. However, it was noted that there were other coliforms present on the petrifilm. Consumers should be mindful that hand sanitizers does not necessarily kill all types of pathogens. Thus, gel hand sanitizers should only be used as an alternative to warm water and soap when there is no other option.

\section{Acknowledgements}

The author thanks Helen Heacock and BCIT Food Technology Laboratory (Ken Keilbart, Melinda Lee, and Erin Frisesen) for providing support and guidance for this study. This study has been funded by BCIT.

\section{References}

Babeluk, R., Jutz, S., Mertlitz, S., Matiasek, J., \& Klaus, C. (2014). Hand Hygiene Evaluation of Three Disinfectant Hand Sanitizers in a Community Setting. PLoS ONE, 9(11), e111969.

http://doi.org/10.1371/journal.pone.0111969

Barratt, P. (2015). Six ways to stop the spread of infections at work. Occupational Health, 67 (3).

British Columbia Centre for Disease Control. (2016). Hand Hygiene. Retrieved from http://www.bccdc.ca/prevention/HandHygiene /default.htm
Centers for Disease Control and Prevention. (2015). Handwashing. Show me the science when to use hand sanitizer. Retrieved from http://www.cdc.gov/handwashing/show-methe- science-hand-sanitizer.html

Choi, J. (2007). Liquid or Foam: A Study of the Comparative Effectiveness of Liquid Soap Versus Foam Soap. British Columbia Institute of Technology

Edmonds, S., Macinga, D., Mays-Suko, P., Duley, C., \& Arbogast, J. (2011). Meeting global standards for hand sanitizer efficacy: formulation matters. BMC Proceedings, 5(Suppl6), P26. http://doi.org/10.1186/17536561-5-S6-P26

Edmonds, S., Mccormack, R., Zhou, S., Macinga, D., \& Fricker, C. (2012). Hand Hygiene Regimens for the Reduction of Risk in Food Service Environments. J Food Prot Journal of Food Protection, 1303-1309.

Heacock, H. \& Karakilic, V. (2015a). Research method: Analysis of Variance: ANOVA. Lecture conducted from British Columbia Institute of Technology, Burnaby.

Heacock, H. \& Karakilic, V. (2015b). Research method module 5: descriptive statistics. Lecture conducted from British Columbia Institute of Technology, Burnaby.

Heacock, H. \& Karakilic, V. (2015c).

Research method module 5: inferential statistics. Lecture conducted from British Columbia Institute of Technology, Burnaby.

Hygiena. (2015). E.coli. MicroSnap. Retrieved from http://www.hygiena.com/microsnap-ecoli- food-and-beverage.html

Kampf, G. \& Kramer, A. (2004). Epidemiologic Background of Hand Hygiene and Evaluation of the Most Important Agents for Scrubs and Rubs. Retrieved from cmr.asm.org/content/17/4/863.full 
Kampf, G., Marschall, S., Eggerstedt, S., \& Ostermeyer, C. (2010). Efficacy of ethanolbased hand foams using clinically relevant amounts: a cross-over controlled study among healthy volunteers. BMC Infectious Diseases, 10, 78. http://doi.org/10.1186/1471-2334- 1078

Keilbart, Ken. (January 20, 2016). Interview British Columbia Institute of Technology.

Luby, S., Kadir, M., Sharker, M., Yeasmin, F., Unicomb, L., \& Islam, M. (2010). A community- randomised controlled trial promoting waterless hand sanitizer and handwashing with soap, Dhaka, Bangladesh.Tropical Medicine \& International Health, 1508-1516.

Microsoft Office Excel. (2013). Microsoft excel. Retrieved from https://products.office.com/en- CA/

National Kidney Foundation. (2015). Hemolytic Uremic Syndrome. Retrieved from https://www.kidney.org/atoz/content/hemolyti c

NCSS. (2015). Statistical \& power analysis software. Retrieved from http://www.ncss.com/

One Step. (2016). The Importance of Hand Sanitizers. Retrieved from http://www.onestepsani.com/health.html

Patel, S. (2004). The efficacy of alcohol-based hand disinfectants products. Nursing Times; 100: 23, 32-34.

Pickering, A., Davis, J., Blum, A. G., Scalmanini, J., Oyier, B., Okoth, G., \& Ram, P. K. (2013). Access to Waterless Hand Sanitizer Improves Student Hand Hygiene Behavior in Primary Schools in Nairobi, Kenya. The American Journal of Tropical Medicine and Hygiene, 89(3), 411-418. http://doi.org/10.4269/ajtmh.13-0008
Pickering, A., Davis, J., \& Boehm, A. (2011). Efficacy of alcohol-based hand sanitizer on hands soiled with dirt and cooking oil. Journal of Water and Health, 429-429.

Purell. (2015). Illness outbreak frequently asked questions. Retrieved from http://www.purell.com

Reynolds, S. A., Levy, F., \& Walker, E. S. (2006). Hand Sanitizer Alert.Emerging Infectious Diseases, 12(3), 527-529. http://doi.org/10.3201/eid1203.050955

SafeHands. (2015). Alcohol Free safeHands sanitizer. Frequently asked questions. Retrieved from https://safehands.com/faqs/

Vidyashankar, S., Varma, S., Patki, P.S. (2011). Hand Sanitizers. Current Science, 100 (11), p1605.

Widmer, A. (2000). Replace Hand Washing with Use of a Waterless Alcohol Hand Rub? Clinical Infectious Diseases, 136-143.

World Health Organization. (2009). WHO Guidelines on Hand Hygiene in Health Care. Retrieved from http://apps.who.int/iris/bitstream/10665/44102 /1/9789241597906 eng.pdf

World Health Organization. (2013). Evidence of hand hygiene to reduce transmission and infections by multidrug resistant organisms in health-care settings. Retrieved from http://www.who.int/gpsc/5may/MDRO_literat ure-review.pdf

World Health Organization. (2016). Enterohaemorrhagic Escherichia coli (ehec). Retrieved from http://www.who.int/mediacentre/factsheets/fs1 $25 / \mathrm{en} /$

Yip, Sophia. (2003). One StepTM Hand Sanitizer vs CAREX R Liquid Soap with 
Hand Washing. British Columbia Institute of Technology 